\title{
Antibody test for Legionella pneumophila detection
}

\author{
Noemí Párraga-Niño a,b, Sara Quero a , Naroa Uria ${ }^{\text {c }}$, Oscar Castillo-Fernandez ${ }^{\text {, }}$, Josune Jimenez-Ezenarro ${ }^{\text {, }}$, \\ Francesc-Xavier Muñoz c, Miquel Sabrià a,b,d,*, Marian Garcia-Nuñez ${ }^{\text {a,b }}$ \\ a Unitat de Malalties Infeccioses, Fundació Institut d'Investigació Germans Trias I Pujol, Badalona, Spain E-08916 \\ b CIBER de Enfermedades Respiratorias, Madrid, Spain E-28029 \\ c Institute of Microelectronics of Barcelona IMB-CNM (CSIC), Cerdanyola del Vallès, Spain E-08193 \\ d Universitat Autònoma de Barcelona, Cerdanyola del Vallès, Spain E-08193
}

\section{A R T I C L E I N F O}

Article history:

Received 29 August 2017

Received in revised form 3 November 2017

Accepted 4 November 2017

Available online 10 November 2017

\section{Keywords:}

Legionella

Antibody

Detection

Cross-reactivity

\begin{abstract}
A B S T R A C T
Legionella pneumophila is responsible for Legionnaires' disease (LD). Its detection in both environmental and clinical samples is mainly performed by culture plate method which requires up to 10 days to obtain results. Nowadays, there are commercial antibodies against this bacterium, but they have not been tested against all subgroups of $L$. pneumophila sg 1 or serogroups 1-16 or their cross-reactions with other non-Legionella bacteria. Indeed, many of these antibodies became available when only 8 serogroups of $L$. pneumophila had been described. We tested 7 antibodies and found that 2 (Mab 8/5 and OBT) specifically detected all the subgroups of L. pneumophila sg 1, one without cross-reactions (Mab8/5). Moreover, the LP3IIG2 antibody detected almost all serogroups tested with lower rates of cross-reactivity, resulting in a specific sensitive antibody for the detection of $L$. pneumophila. LP3IIG2 presented higher rate of cross-reactivity against respiratory non-Legionella isolates, thereby contraindicating its clinical applicability.
\end{abstract}

(c) 2017 Elsevier Inc. All rights reserved.

\section{Introduction}

Legionella is a Gram-negative bacterium ubiquitous in fresh water habitats (Null, 1996). Legionella can colonize man-made water systems and be dispersed by aerosols generated by showers, faucets, cooling towers, whirlpool spas, and fountains. The inhalation and microaspiration of Legionella (Sabria and Yu, 2002) by susceptible people can cause Legionnaire's disease (LD), which is manifested as pneumonia, presenting a case fatality rate of 10\% (http://ecdc.europa.eu/en/ publications/Publications/legionnaires-disease-2015.pdf), or a mild nonpneumonic febrile illness called Pontiac fever (Kaufmann et al., 1981). Legionella pneumophila contains at least 16 serogroups, but serogroup 1 ( $\mathrm{sg} \mathrm{1}$ ) accounts for the majority of European and American clinical isolates (Yu et al., 2002).

Urinary antigen test is the gold standard to detect $L$. pneumophila infections due to the speed and simplicity of this test. However, it only detects $L$. pneumophila sg 1 cases, producing a diagnostic blind spot for LD caused by L. pneumophila non-sg 1 and Legionella spp. Although more diagnostic tools are available, only 5 immunodiagnostic methods (Alere BinaxNOW® Legionella; http://www.alere.com/en/home/product-details/binaxnow-legionella-urinary-antigen-eia.html; http:// www.meridianbioscience.com/diagnostic-products/respiratory/tru/ tru-legionella.aspx; http://www.sascientific.com/news-events/8-news/

\footnotetext{
* Corresponding author. Tel.: +34-934-978-680; fax: +34-934-978-654.

E-mail address: msabria.germanstrias@gencat.cat (M. Sabrià).
}

5-legionella-test; http://www.trinitybiotech.com/products/legionellaurinary-antigen-eia/) have been approved for commercial purposes by the US Food and Drug Administration (http://www.fda.gov/).

Legionella genus-specific (Helbig et al., 1995b; Steinmetz et al., 1991 ) and L. pneumophila species-specific (Goldstein and Gosting; Gosting et al., 1984; Helbig et al., 1995b; Hindahl and Iglewski, 1986; Rockey et al., 1996) antibodies were described and evaluated decades ago. Since then, 8 new serogroups have been described, and a larger number of antibodies have been commercialized without much information about the specificity.

The objective of this study was to determine the specificity of 7 antibodies against L. pneumophila (subgroups of sg 1 and serogroups), Legionella species, and cross-reactions. Thus, the use of an already validated antibody is the most adequate way to achieve the specificity of all $L$. pneumophila serogroups to be applied in an environmental and/ or clinical detection tool.

\section{Materials and Methods}

\subsection{Bacterial Isolates}

We used 79 L. pneumophila and non-L. pneumophila bacterial isolates obtained from different sources: American Type Culture Collection (ATCC), environmental water samples (E), and respiratory samples (R). Legionella microorganisms were grown on BCYE for $72 \mathrm{~h}$, and non-Legionella environmental isolates were grown on Tryptic Soy Agar 
(environmental isolates) or chocolate agar for non-Legionella respiratory isolates for 24 to $72 \mathrm{~h}$.

Bacterial characterization was performed by 165 rRNA gene amplification and sequencing using the universal primers E27 forward (AGAGTTTGATCMTGGCTCAG) and B530 reverse (CCGCGGCKGCTGG CAC). Furthermore, Legionella species were identified by amplification and sequencing of the mip gene (Ratcliff et al., 1998). Sequencing was performed by the Genomics Core Facility at the Germans Trias i Pujol Research Institute.

\subsection{Antibodies}

Seven antibodies were tested: Mab 8/5 (Dresden Panel), Mip 1 (MBS855187, MyBiosource, San Diego, CA), Mip 2 (MBS852400, MyBiosource), Omp 28 (MBS852640, MyBiosource), LP3IIG2 (ATCC HB-8472), OBT (OBT0943P, AbDserotec, Bio-Rad, Hercules, CA), BacTrace (01-90-03, SeraCare, Milford, MA). Secondary antibodies were used with nonconjugated antibodies. Anti-mouse conjugated with horseradish peroxidase (HRP) (ab97265, Abcam, Cambridge, UK) was used to detect Mab 8/5, Mip 1, Mip 2, and Omp 28; anti-rabbit HRP (ab97200, Abcam) was used to detect KPL; and anti-mouse conjugated with fluorescein-5-isothiocyanate (FITC) (F0257, Sigma-Aldrich Co., St. Louis, MO) was used in the immunofluorescence assay (IFA) test.

LP3IIG2 was obtained from the hybrid cell line HB-8472 (ATCC). This hybridoma was cultured, purified, and HRP conjugated by the Antibody Service of Universitat Autònoma of Barcelona.

\subsection{Adjusting Antibody Concentration}

The working concentration of each antibody was determined using an isolate of L. pneumophila sg. 1 subgroup Philadelphia (reference isolate) according to the protocol described below.

\subsection{Antibody Assay}

The antibodies were screened with an in-house enzyme-linked immunosorbent assay (ELISA) test using Legionella and non-Legionella isolates as a capturing antigen. Bacterial suspensions were prepared in phosphate-buffered saline (PBS) pH 7.2 and adjusted to an optical density (OD) of 0.3 at a wavelength of $625 \mathrm{~nm}\left(\sim 10^{8} \mathrm{CFU} / \mathrm{mL}\right)$. One hundred microliters of these bacterial suspensions was added to a 96-well plate (Nunc Brand, Denmark) and was dried overnight at $40^{\circ} \mathrm{C}$. These plates were stored dried at $4{ }^{\circ} \mathrm{C}$ until use. The plates were blocked with $10 \%$ fetal bovine serum in PBS for $2 \mathrm{~h}$ at $37^{\circ} \mathrm{C}$. One hundred microliters of the primary antibody was added to each well and incubated for $1 \mathrm{~h}$ at $37^{\circ} \mathrm{C}$. Then, the plates were washed three times with $0.05 \%$ Tween 20 in PBS (PBS-Tween). For nonconjugated antibodies, the plates were incubated with a secondary HRP-conjugated antibody for $1 \mathrm{~h}$ at $37^{\circ} \mathrm{C}$ and washed 3 times with PBS-Tween. Developing reaction was performed with $50 \mu \mathrm{L}$ 3,3',5,5'-Tetramethylbenzidine (TMB, Sigma) during 20 min at room temperature in the dark. The reaction was stopped with $50 \mu \mathrm{L}$ of $1 \mathrm{~N} \mathrm{H}_{2} \mathrm{SO}_{4}$, and the colorimetric reaction was measured at OD $450 \mathrm{~nm}$ using a Varioskan Flash (Thermo Electron Corporation).

Each test was performed in duplicate in the experiment and in triplicate on different days.

\subsection{Indirect Immunofluorescence Assay}

Bacterial suspensions were prepared in PBS or in $1 \%$ formalin and adjusted to an OD of 0.3 at a wavelength of $625 \mathrm{~nm}$, and employed as antigen for indirect IFA. LP3IIG2 was used as a primary antibody and the anti-mouse FITC as a secondary antibody. Results were observed in a Fluorescence Microscope (Axioscope, Zeiss, Germany) and were classified as positive if they reacted strongly.

\subsection{Data Analysis}

The ELISA background value was calculated for each isolate according to the antibody used. For primary conjugated antibodies, the background value was determined as the signal obtained from the antibody without bacteria. For antibodies that required a secondary antibody, the background value was determined as the signal obtained from the bacteria with secondary antibody, without the primary antibody. After the subtraction of the background value, all the results were normalized using the value obtained with the reference isolate included in each experiment as an internal control. The detection limit was established for each antibody as the mean $(\bar{X})$ plus 3 times the standard deviation (SD) obtained in the background test: Mab 8/5 (0.25), Mip 1 (0.25), Mip 2 (0.25), Omp 28 (0.25), LP3IIG2 (0.19), Bac-Trace $(0.5)$, and OBT (0.11). Results were expressed as positive $(+)$, negative $(-)$, or inconclusive $(-/+)$ (discrepancies among replicates). OD values (mean and SD) were shown in Supplementary material.

The study was performed in 2 different steps. Seven antibodies were first evaluated at a working concentration according to the protocol described. The antibodies were screened in this first trial, and according to the results obtained, the test was repeated increasing the number of isolates tested.

\section{Results}

\subsection{Antibody Assay}

The reactivity of 7 antibodies was tested to 19 Legionella isolates and 14 non-Legionella isolates (Table 1). First, the antibodies were checked to select only those that reacted with $L$. pneumophila isolates, discarding the non-specific L. pneumophila antibodies.

The Mab 8/5 (1:200) was described to exclusively recognize L. pneumophila sg 1 as described elsewhere (Helbig et al., 1995a), and it did not present any cross-reaction.

The 2 monoclonal antibodies against the Mip protein (Mip 1: $5 \mathrm{ng} / \mu \mathrm{L}$ and Mip 2: $10 \mathrm{ng} / \mu \mathrm{L}$ ) did not react against some $L$. pneumophila serogroups or some subgroups of L. pneumophila sg. 1. Furthermore, Mip 2 showed even less reactivity against the L. pneumophila serogroups than Mip 1 (Table 1).

The monoclonal Omp 28 antibody ( $5 \mathrm{ng} / \mu \mathrm{L}$ ) showed high reactivity to almost all the isolates tested (L. pneumophila and non-Legionella), with higher values in non-Legionella isolates.

The polyclonal OBT antibody $(20 \mathrm{pg} / \mu \mathrm{L})$ reacted with all the isolates of $L$. pneumophila sg 1 and showed reactivity with $L$. pneumophila sg 13 and some cross-reactions with environmental isolates.

The polyclonal Bac-Trace antibody was adjusted to $2 \mathrm{pg} / \mu \mathrm{L}$. This concentration was lower compared to the other antibodies. Bac-Trace showed a low rate of positive results against Legionella isolates (L. pneumophila sg 1 Philadelphia, L. pneumophila sg 9 and sg 15) and cross-reacted against 7 out of 14 environmental isolates.

The LP3IIG2 antibody ( $2.5 \mathrm{ng} / \mu \mathrm{L})$ showed specificity against all the serogroups of $L$. pneumophila and cross-reactions against $L$. micdadei and Sphingomonas spp.

Only 2 of the 7 antibodies tested, OBT and LP3IIG2, were selected to test their reactivity against 47 more isolates (26 Legionella and 21 nonLegionella environmental and respiratory isolates) (Table 2). We chose LP3IIG2 due to their sensitivity in front of all L. pneumophila serogroups tested. OBT and Mab 8/5 showed a specific recognition of $L$. pneumophila sg 1, but OBT showed incongruent results regarding previous publications (Yanez et al., 2005), so we decided to carry out the second step in the ELISA tests also with this antibody to characterize it.

The LP3IIG2 antibody reacted against all the subgroups of L. pneumophila sg 1 (except the 2 Benidorm and 1 Camperdown isolates) and 1 isolate of L. pneumophila sg 4, 6, 8, and 10 (Table 2). Regarding the $L$. non-pneumophila isolates, this antibody only recognized L. micdadei. Testing the environmental non-Legionella isolates, this 
Table 1

Reactivity in the first step of the ELISA test with 7 L. pneumophila antibodies (+ for positive results, - for negative results, and -/+ for discrepancy between replicates).

\begin{tabular}{|c|c|c|c|c|c|c|c|c|}
\hline Isolates (ref) ${ }^{a}$ & Source $^{\mathrm{b}}$ & $\begin{array}{l}\text { Mab 8/5 } \\
(1 / 200)^{\mathrm{C}}\end{array}$ & $\begin{array}{l}\text { Mip } 1 \\
(5 \mathrm{ng} / \mu \mathrm{L})^{\mathrm{c}}\end{array}$ & $\begin{array}{l}\text { Mip } 2 \\
(10 \mathrm{ng} / \mu \mathrm{L})^{\mathrm{c}}\end{array}$ & $\begin{array}{l}\text { Omp } 28 \\
(5 \mathrm{ng} / \mu \mathrm{L})^{\mathrm{c}}\end{array}$ & $\begin{array}{l}\text { LP3IIG2 } \\
(2.5 \mathrm{ng} / \mu \mathrm{L})^{\mathrm{c}}\end{array}$ & $\begin{array}{l}\text { Bac-Trace } \\
(2 \mathrm{pg} / \mu \mathrm{L})^{\mathrm{c}}\end{array}$ & $\begin{array}{l}\text { OBT } \\
(20 \mathrm{pg} / \mu \mathrm{L})^{\mathrm{C}}\end{array}$ \\
\hline \multicolumn{9}{|l|}{ Legionella isolates } \\
\hline Lp 1 Philadelphia (A) & $\mathrm{E}$ & + & + & + & + & + & + & + \\
\hline Lp 1 France $(\mathrm{A})$ & $\mathrm{E}$ & + & + & - & + & + & - & + \\
\hline Lp 1 OLDA(A) & $\mathrm{E}$ & + & - & - & + & + & - & + \\
\hline Lp 1 Oxford (A) & $\mathrm{E}$ & + & + & + & + & + & - & + \\
\hline Lp 3 & $\mathrm{E}$ & - & - & - & + & + & - & - \\
\hline $\operatorname{Lp} 8(\mathrm{~A})$ & $\mathrm{E}$ & - & - & + & + & - & - & - \\
\hline $\operatorname{Lp} 9(\mathrm{~A})$ & $\mathrm{E}$ & - & + & + & + & + & + & - \\
\hline Lp 10 (B) & 43283 & - & + & - & + & + & - & - \\
\hline Lp 11 & 43130 & - & - & - & - & + & - & - \\
\hline Lp $12(\mathrm{~A})$ & $\mathrm{E}$ & - & + & + & + & $-/+$ & - & - \\
\hline Lp 12 (B) & 43290 & - & + & - & + & + & - & - \\
\hline Lp 13 & 43736 & - & + & - & + & + & - & $-/+$ \\
\hline Lp 14 (B) & 43703 & - & + & - & + & + & - & - \\
\hline Lp 15 & E & - & + & - & + & + & + & - \\
\hline L. micdadei & $\mathrm{E}$ & - & + & + & + & + & + & + \\
\hline L. parisiensis (A) & $\mathrm{E}$ & - & - & + & + & - & + & - \\
\hline L. anisa & 35292 & - & + & - & + & - & - & - \\
\hline L. dumoffi & 35850 & - & + & + & + & - & - & $-/+$ \\
\hline L. gormani & 33297 & - & + & - & + & - & + & - \\
\hline \multicolumn{9}{|l|}{ Non-Legionella isolates } \\
\hline Aeromonas veronii & $\mathrm{E}$ & - & + & + & + & - & + & $-/+$ \\
\hline Sphingomonas spp. & $\mathrm{E}$ & - & + & + & + & + & + & - \\
\hline Delftia spp. & $\mathrm{E}$ & - & + & + & + & - & + & $-/+$ \\
\hline Stenotrophomonas acidanimiphila & $\mathrm{E}$ & - & + & + & + & - & - & + \\
\hline Paenibacillus dendritiformis & $\mathrm{E}$ & - & - & + & + & - & - & - \\
\hline Ochrobactrum & $\mathrm{E}$ & - & + & - & + & - & - & - \\
\hline Bacillus spp. (A) & $\mathrm{E}$ & - & - & - & + & - & + & - \\
\hline Bacillus spp. (B) & $\mathrm{E}$ & - & + & + & + & - & + & - \\
\hline Bacillus spp. (C) & $\mathrm{E}$ & - & - & - & + & - & - & - \\
\hline Pseudomonas aeruginosa (A) & $\mathrm{E}$ & - & + & + & + & - & + & - \\
\hline Chryoseobacterium spp. & $\mathrm{E}$ & - & - & - & - & $-/+$ & - & - \\
\hline Bacillus licheniformis & $\mathrm{E}$ & - & - & + & + & - & - & - \\
\hline Serratia spp. & $\mathrm{E}$ & - & - & - & + & - & + & - \\
\hline Acetinobacter spp. & $\mathrm{E}$ & - & - & + & + & - & - & - \\
\hline
\end{tabular}

Lp = Legionella pneumophila

a Indicates the local laboratory number in order to differentiate isolates.

b $E=$ environmental water source; reference number for ATCC source.

c Concentration of the antibody.

antibody only cross-reacted with Sphingomonas spp., and an inconclusive result was obtained with Chryoseobacterium spp. (positive and negative results in the replicates). LP3IIG2 showed high cross-reactivity against 9 out of 20 respiratory isolates belonging to 4 different bacterial species (Staphylococcus aureus, Neisseria spp., Moraxella catharralis, and Haemophilus parainfluenzae).

The OBT antibody showed a high specificity versus all the subgroups of $L$. pneumophila sg 1 (Table 2). OBT cross-reacted against $L$. micdadei, with inconclusive results against $L$. pneumophila sg 13 and $L$. dumoffi. Testing the environmental non-Legionella isolates, this antibody only cross-reacted with Stenotrophomonas acidanimiphila, and inconclusive results were obtained with Aeromonas veronii and Delftia spp. OBT showed cross-reactivity with 7 respiratory isolates belonging to 3 different bacterial species (Staphylococcus aureus, Neisseria spp., and Moraxella catharralis).

\subsection{Indirect Immunofluorescence Assay}

All the $L$. pneumophila isolates $(n=11)$ with negative result in the LP3IIG2 antibody assay were confirmed as negative by IFA (Fig. 1). Results obtained in IFA test showed no differences between samples prepared in PBS or formalin.

\section{Discussion}

In the last 30 years, the number of $L$. pneumophila serogroups has doubled, and the number of commercialized antibodies has exponentially risen. However, the information available about their reactivity is limited (reactivity against Legionella and other cohabitant species). It is necessary to make efforts to validate the reactivity of these antibodies to establish which ones are specific to L. pneumophila serogroup 1 , to L. pneumophila non-sg 1 , and to Legionella nonpneumophila and cross-reactivity with other bacterial species.

The antibodies tested in this study did not present a high specificity and sensitivity to L. pneumophila. However, 3 antibodies showed promising results. Mab 8/5 and OBT showed reactivity to L. pneumophila sg 1, although Mab 8/5 had higher specificity (it did not present any cross-reaction). Mab $8 / 5$ recognized the lipopolysaccharide (LPS), the main conserved domain in L. pneumophila sg 1 (Helbig et al., 1995a). On the other hand, the OBT also presented this sensitivity to L. pneumophila sg 1, albeit with a lower specificity, because it also reacted with some non-Legionella isolates.

LP3IIG2 was designed against the mompS protein and showed the best sensitivity/specificity ratio. Fluorescein-conjugated LP3IIG2 antibody is commercially available, and it detects almost all the serogroups. However, contrary to a previous study (Goldstein and Gosting), it did not react with some of the isolates belonging to different L. pneumophila serogroups $(1,4,6,8$, and 10$)$ or with some respiratory isolates. It is important to note the variability observed in the results obtained with isolates of the same serogroup. These discrepancies observed were confirmed with another technique (IFA) using 2 sample preparations. Membrane changes due to treatment with formalin did not improve the results obtained in the IFA.

LP3IIG2 was the most sensitive antibody tested against L. pneumophila. Moreover, negative results obtained with 
Table 2

Reactivity in the second step of the ELISA test with the LP3IIG2 and OBT antibody versus Legionella, environmental and respiratory isolates ( + for positive results, - for negative results and $-/+$ for discrepancy between replicates).

\begin{tabular}{|c|c|c|c|c|}
\hline Isolates (ref) ${ }^{\mathrm{a}}$ & Number isolates & Source $^{\mathrm{b}}$ & $\begin{array}{c}\text { LP3IIG2 } \\
(2.5 \mathrm{ng} / \mu \mathrm{L})^{\mathrm{c}}\end{array}$ & $\begin{array}{c}\text { OBT } \\
(20 \mathrm{pg} / \mu \mathrm{L})^{\mathrm{c}}\end{array}$ \\
\hline \multicolumn{5}{|l|}{ Legionella isolates } \\
\hline Lp 1 Knoxville & 2 & $\mathrm{E}$ & + & + \\
\hline Lp 1 Philadelphia & 2 & $\mathrm{E}$ & + & + \\
\hline Lp 1 Benidorm & 2 & $\mathrm{E}$ & - & + \\
\hline Lp 1 France (A) & 1 & $\mathrm{E}$ & + & + \\
\hline Lp 1 France (B) & 1 & $\mathrm{E}$ & $-/+$ & + \\
\hline Lp 1 OLDA & 2 & $\mathrm{E}$ & + & + \\
\hline Lp 1 Oxford & 2 & $\mathrm{E}$ & + & + \\
\hline Lp 1 Bellingham & 2 & $\mathrm{E}$ & + & + \\
\hline Lp 1 Heysham & 2 & $\mathrm{E}$ & + & + \\
\hline Lp 1 Camperdown (A) & 1 & $\mathrm{E}$ & $-/+$ & + \\
\hline Lp 1 Camperdown (B) & 1 & $\mathrm{E}$ & - & + \\
\hline $\operatorname{Lp} 3$ & 1 & $\mathrm{E}$ & + & - \\
\hline Lp 3 ST93 & 1 & $\mathrm{E}$ & $-/+$ & - \\
\hline $\operatorname{Lp} 4(\mathrm{~A})$ & 1 & $\mathrm{E}$ & - & - \\
\hline Lp $4 \mathrm{P}$ & 1 & $\mathrm{E}$ & + & - \\
\hline Lp 6 & 1 & $\mathrm{E}$ & - & - \\
\hline $\operatorname{Lp} 8(\mathrm{~A}, \mathrm{C})$ & 2 & $\mathrm{E}$ & - & - \\
\hline Lp 8 (B) & 1 & $\mathrm{E}$ & + & - \\
\hline Lp 9 & 3 & $\mathrm{E}$ & + & - \\
\hline Lp $10(\mathrm{~A})$ & 1 & $\mathrm{E}$ & - & - \\
\hline Lp 10 (B) & 1 & 43283 & + & - \\
\hline Lp 11 & 1 & 43130 & + & - \\
\hline Lp $12(\mathrm{~A})$ & 1 & $\mathrm{E}$ & $-/+$ & - \\
\hline Lp 12 (B) & 1 & 43290 & + & - \\
\hline Lp 13 & 1 & 43736 & + & $-/+$ \\
\hline Lp $14(\mathrm{~A})$ & 1 & $\mathrm{E}$ & $-/+$ & - \\
\hline Lp 14 (B) & 1 & 43703 & + & - \\
\hline Lp 15 & 1 & $\mathrm{E}$ & + & - \\
\hline L. micdadei & 1 & $\mathrm{E}$ & + & + \\
\hline L. parisiensis & 3 & $\mathrm{E}$ & - & - \\
\hline L. anisa & 1 & 35292 & - & - \\
\hline L. dumoffi & 1 & 35850 & - & $-/+$ \\
\hline L. gormani & 1 & 33297 & - & - \\
\hline
\end{tabular}

Lp = Legionella pneumophila.

a Indicates the local laboratory number in order to differentiate isolates.

b $E=$ environmental water source; $R=$ respiratory source; reference number for ATCC source.

c Concentration of the antibody.

L. pneumophila sg 1 isolates could be improved by combination with a specific L. pneumophila sg 1 antibody such as Mab 8/5. The LP3IIG2 antibody would only be useful for environmental detection if a thorough validation is performed because it only presented 1 clear crossreaction (Sphingomonas spp.). Regarding clinical detection, neither LP3IIG2 nor OBT would be suitable because of the low specificity of these antibodies.

This work highlights the relevance of the need for validation studies. Indeed, a large number of antibodies are now available in the market, but information regarding the reactivity of most of these antibodies is limited. Several antibodies against different proteins (mip, momps,
Hsp60) have shown to be Legionella genus or L. pneumophila species specific (Gosting et al., 1984; Helbig et al., 1995b; Hindahl and Iglewski, 1986). However, most of these antibodies are not yet commercially available. In this respect, several antibodies against the previously described proteins have been produced, with the manufacturer only performing the validation step at protein recognition and not to differential epitope recognition. Three of the antibodies ruled out in the present study corresponded to this type of antibody (Mip1, Mip2, and Omp 28).

These results reinforce the importance of a good design in the production of antibodies. A protein may contain a species-specific epitope, but if the antibody produced is not specifically designed against this epitope rather than against the whole protein, we may obtain antibodies that are not species specific, having a good sensitivity but not specificity, hence the importance of a validation process after the production of antibodies.

Other specific antibodies have been described. However, they present some caveats. A genus-specific epitope was described on the heat shock protein (Steinmetz et al., 1991). Nonetheless, the expression of this epitope was lower in the membrane, making it unsuitable for our experiment. The 53-3D12 antibody (Barthe et al., 1988) has been described to specifically recognize a common epitope on the LPS of L. pneumophila sg $1-8$, but this antibody is not commercially available, and information about its cross-reactivity is lacking.

The major limitation of this study is that the results of the validation cannot be extrapolated to other immunological techniques, and additional cross-reactions cannot be ruled out.

In conclusion, in the present study, we evaluated several antibodies for rapid identification of L. pneumophila, but only 2 (Mab 8/5 and OBT) are suitable for the specific recognition of L. pneumophila sg 1 and only 1 (LP3IIG2) for the specific recognition of all the serogroups of L. pneumophila. LP3IIG2 shows compatible results to be applied in the specific L. pneumophila detection in water samples. This antibody can be applied in different methods to detect L. pneumophila (Yamaguchi et al., 2017; Yanez et al., 2005), improving specificity of the method. Continuing development by sourcing further monoclonal antibodies to increase the range of detection, automatization, and further optimization will improve the detection.

The prevention of legionellosis is a public health issue and requires specific systems to control and detect these microorganisms. The discovery of an antibody to specifically detect $L$. pneumophila would open the door to the development of a simple rapid detection system. If the antigen recognized is surface exposed, the protocol would be simplified by the absence of sample manipulation, detecting intact cells. In the coming years, the goal to reduce legionellosis is to minimize disease transmission through risk assessment, regular maintenance of potable and nonpotable water systems, and water monitoring and treatment in facilities that care for susceptible populations (Sehulster et al., 2003; Tablan et al., 2004).

The discovery of a specific sensitive antibody/cocktail antibody to all L. pneumophila serogroups would allow the development of a specific automated device for the detection and comprehensive surveillance of
A

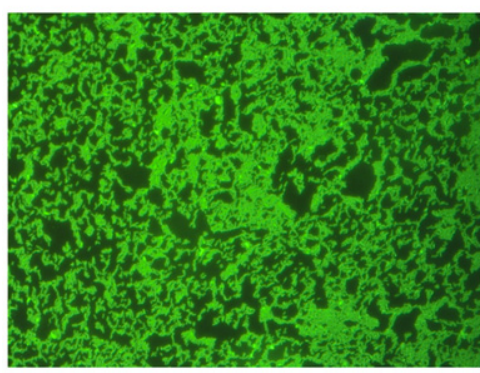

B

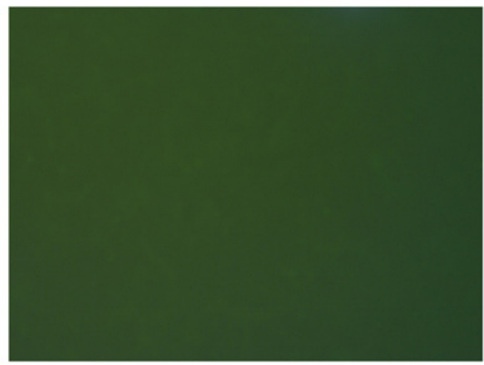

C

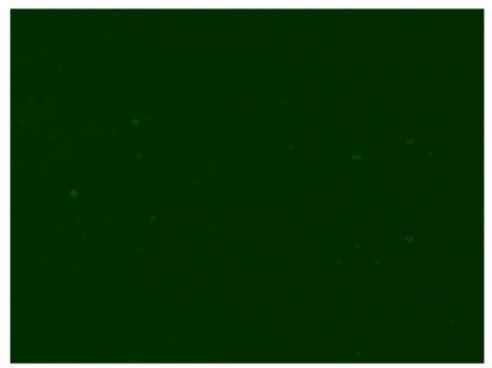

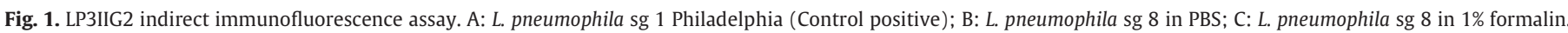
L. pneumophila sg 8 is shown as an example of the results obtained with all the isolates tested (isolates that showed negative result with the LP3IIG 2 antibody in the antibody assay). 
L. pneumophila in water facilities. Moreover, the implementation of a system based on an antibody would reduce the variability in the analyses and also avoid the nonidentification of viable but not culturable cells.

\section{Acknowledgments}

The authors acknowledge the sponsorship by Gas Natural Fenosa and financial support from CIBER and CERCA Programme/Generalitat de Catalunya. N.U. (TECSPR15-1-0046) and O.C (TECSPR14-2-0034) have received funding from the People Programme (Marie Curie Actions) of the 7th Framework Programme of the European Union (FP7/2007-2013), TECNIOspring program from the Agency for Business Competitiveness of the Government of Catalonia (ACCIÓ). The authors thanks the collaboration of Aqualab SL in the obtaining of bacterial species from water samples.

\section{Appendix A. Supplementary data}

Supplementary data to this article can be found online at https://doi. org/10.1016/j.diagmicrobio.2017.11.005.

\section{References}

Alere BinaxNOW® Legionella. http://www.alere.com/es/home/product-details/ binaxnow-legionella.html.

Barthe C, Joly JR, Ramsay D, Boissinot M, Benhamou N. Common epitope on the lipopolysaccharide of Legionella pneumophila recognized by a monoclonal antibody. J Clin Microbiol 1988;26(5):1016-23.

Goldstein Lynn C, Gosting Larry H. Method and composition for the detection and diagnosis of Legionella pneumophila; 1984. US $4851333 \mathrm{~A}$.

Gosting LH, Cabrian K, Sturge JC, Goldstein LC. Identification of a species-specific antigen in legionella pneumophila by a monoclonal antibody. J Clin Microbiol 1984;20(6):1031-5.

Helbig JH, Lück PC, Knirel YA, Witzleb W, Zähringer U. Molecular characterization of a virulence-associated epitope on the lipopolysaccharide of legionella pneumophila serogroup 1. Epidemiol Infect 1995a;115(1):71-8.

Helbig JH, Ludwig B, Lück PC, Groh A, Witzleb W, Hacker J. Monoclonal antibodies to Legionella Mip proteins recognize genus- and species-specific epitopes. Clin Diagn Lab Immunol 1995b;2(2):160-5.
Hindahl MS, Iglewski BH. Outer membrane proteins from legionella pneumophila serogroups and other legionella species. Infect Immun 1986;51(1):94-101.

http://ecdc.europa.eu/en/publications/Publications/legionnaires-disease-2015.pdf.

http://www.alere.com/en/home/product-details/binaxnow-legionella-urinary-antigeneia.html.

http://www.fda.gov/.

http://www.meridianbioscience.com/diagnostic-products/respiratory/tru/tru-legionella. aspx.

http://www.sascientific.com/news-events/8-news/5-legionella-test.

http://www.trinitybiotech.com/products/legionella-urinary-antigen-eia/.

Kaufmann AF, McDade JE, Patton CM, Bennett JV, Skaliy P, Feeley JC, et al. Pontiac fever: isolation of the etiologic agent (Legionella pneumophilia) and demonstration of its mode of transmission. Am J Epidemiol 1981;114(3):337-47.

Fliermans. Ecology of legionella: from data to knowledge with a little wisdom. Microb Ecol 1996;32(2):203-28.

Ratcliff RM, Lanser JA, Manning PA, Heuzenroeder MW. Sequence-based classification scheme for the genus Legionella targeting the mip gene. J Clin Microbiol 1998; 36(6):1560-7.

Rockey DD, Chesebro BB, Heinzen RA, Hackstadt T. A 28 kDa major immunogen of chlamydia psittaci shares identity with Mip proteins of Legionella spp. and Chlamydia trachomatis-cloning and characterization of the C. Psittaci mip-like gene. Microbio Read Engl 1996;142(Pt 4):945-53. https://doi.org/10.1099/00221287-142-4-945.

Sabria M, Yu VL. Hospital-acquired legionellosis: solutions for a preventable infection. Lancet Infect Dis 2002;2(6):368-73.

Sehulster L, RYW Chinn, Centers for Disease Control \& Prevention (CDC), HICPAC. Guidelines for environmental infection control in health-care facilities. Recommendations of CDC and the Healthcare Infection Control Practices Advisory Committee (HICPAC). MMWR Recomm Rep 2003;52(RR-10):1-42.

Steinmetz I, Rheinheimer C, Hübner I, Bitter-Suermann D. Genus-specific epitope on the 60-kilodalton legionella heat shock protein recognized by a monoclonal antibody. J Clin Microbiol 1991;29(2):346-54

Tablan OC, Anderson LJ, Besser R, Bridges C, Hajjeh R, CDC, et al. Guidelines for preventing health-care-associated pneumonia, 2003: recommendations of CDC and the healthcare infection control practices advisory committee. MMWR Recomm Rep 2004;53(RR-3):1-36

Yamaguchi N, Tokunaga Y, Goto S, Fujii Y, Banno F, Edagawa A. Rapid on-site monitoring of Legionella pneumophila in cooling tower water using a portable microfluidic system. Sci Rep 2017;7(1). https://doi.org/10.1038/s41598-017-03293-9.

Yanez MA, Carrasco-Serrano C, Barbera VM, Catalan V. Quantitative detection of legionella pneumophila in water samples by immunomagnetic purification and real-time PCR amplification of the dotA gene. Appl Environ Microbiol 2005;71(7):3433-41. https://doi.org/10.1128/AEM.71.7.3433-3441.2005.

Yu VL, Plouffe JF, Pastoris MC, Sout JE, Schousboe M, Widmer A, et al. Distribution of legionella species and serogroups isolated by culture in patients with sporadic community-acquired legionellosis: an international collaborative survey. J Infect Dis 2002;186(1):127-8. https://doi.org/10.1086/341087. 